\title{
Non-steroidal anti-inflammatory drugs in patients with peptic ulcer disease: rarely justified in terms of cost or patient benefit
}

\author{
Chris Hawkey
}

The prescription of non-steroidal anti-inflammatory drugs is so widespread that all prescribers inevitably face difficult decisions about their use. Decisions related to peptic ulcers are taken on the basis of remarkably little available data.

\section{How dangerous are non-steroidal anti-inflammatory drugs?}

At the extremes of opinion there is an artificial debate between some gastroenterologists who regard non-steroidal anti-inflammatory drugs as an important cause of morbidity and death and some rheumatologists who regard them as free of hazard. These views reflect different facets of risk. Many elderly patients presenting to gastroenterologists with bleeding or perforation are taking non-steroidal anti-inflammatory drugs, which roughly treble the risks. ${ }^{12}$ In absolute terms, however, the risk remains low (around one in 500 patient years ${ }^{3}$ ). None the less, because prescription is so widespread this translates into substantial iatrogenic disease, which would fall with curtailed prescribing.

\section{Do non-steroidal anti-inflammatory drugs retard healing?}

Few controlled data address this important question. Trials tend to be small, to amalgamate data on gastric and duodenal ulcers, and to lack appropriate comparisons. Including small ulcers or (inadvertently) erosions in trials may generate better results than occur in clinical practice. One group has consecutively reported evidence that ulcers associated with aspirin are easy and hard to heal. ${ }^{+5}$ In patients continuing to take non-steroidal anti-inflammatory drugs another study showed that the ulcer healed in six weeks in six out of 13 patients taking placebo and nine out of 14 taking cimetidine." These results have been regarded as reassuring, although the size of the ulcer was not stated and the patients were not compared with patients who were not taking non-steroidal antiinflammatory drugs. In a further trial ranitidine or sucralfate, or both, ultimately healed gastric or duodenal ulcers in $91 \%$ of patients who stopped taking non-steroidal anti-inflammatory drugs (median healing time 4.6 weeks) and $77 \%$ of those who continued (median healing time 5.0 weeks). More recently misoprostol has been shown to enhance healing of gastric ulcers associated with aspirin. ${ }^{8}$

Some ulcers may heal when non-steroidal antiinflammatory drugs continue to be taken, but how many do is not clear.

\section{Are non-steroidal anti-inflammatory drugs dangerous during healing?}

Even if non-steroidal anti-inflammatory drugs do not retard healing they may enhance the risk of complications before healing occurs - for example, by an antihaemostatic mechanism (see below). There are

Department of

Therapeutics, University Hospital, Nottingham NG7 7UH

Chris Hawkey, MRCP, reader in gastroenterology

Br Med f 1989;298:177-8 reporting to the regulatory authorities of adverse gastrointestinal drug reactions. ${ }^{410}$ There may be real differences among the different drugs, " but rates of reporting are so prone to confounding that we cannot be sure. Ibuprofen seems fairly safe for patients who do not need major analgesia.

\section{Can non-steroidal anti-inflammatory drugs be taken} again after ulcers have healed?

Ulcers associated with non-steroidal anti-inflammatory drugs are often silent ${ }^{12}$ so dyspepsia will not necessarily signal a relapse. This observation raises interesting questions. Do non-steroidal anti-inflammatory drugs reduce ulcer pain or do they provoke complications in pre-existing ulcers, having been given to patients with silent but not painful ulcers?

Ulcers relapse after healing. How far non-steroidal anti-inflammatory drugs enhance the risk has not been quantified. Most prescribers would use maintenance treatment, but the supporting evidence is indirect and there is none to guide choosing a dose. In a study of maintenance treatment given to unselected patients with an ulcer for one to nine years gastrointestinal bleeding was uncommon ( $1 \cdot 1 \%$ of compliant patients); some patients were taking non-steroidal anti-inflammatory drugs, but the number was too small to address management in this group specifically. ${ }^{13}$

\section{Should patients who start taking non-steroidal anti-inflammatory drugs receive prophylaxis?}

Whether patients who start taking non-steroidal anti-inflammatory drugs should have prophylaxis is unknown. Several supplementary questions need to be answered.

What is the prophylaxis against? - Failing to suppress erosions ${ }^{14}$ probably does not matter. But are we correct to assume that ulcer development is the target? As well as being ulcerogenic non-steroidal anti-inflammatory drugs interfere with platelet aggregation and prolong the bleeding time ${ }^{15}$; haematemesis often occurs in the short term.' There may be a case for evaluating haemostatic agents for prophylaxis.

What agents should be used? - In practice, many doctors prescribe an $\mathrm{H}_{2}$ receptor antagonist. Although normal doses only partially reduce short term injury, ${ }^{16}$ total acid suppression may abolish $\mathrm{it}^{1-1 \times}$; thus high doses of $\mathrm{H}_{2}$ receptor antagonists or proton pump inhibitors might give better prophylaxis. Antisecretory doses of prostaglandins also prevent short term injury, ${ }^{19} 2^{20}$ but comparative trials with ulcers as end points will be needed before optimum agents and strategies are defined.

How long should prophylaxis be given?-Acute gastric mucosal injury lessens with adaptation to the continued ingestion of non-steroidal anti-inflammatory drugs. ${ }^{21}$ Half of the bleeds from ulcers associated with the drugs occur within three months after treatment is started. Thus in theory there might be a critical period during which prophylaxis is particularly appropriate.

Some practical problems - Two factors militate, however, against universal prophylaxis. Firstly, no regular drug regimen can cope with the inevitable lapses of compliance in symptomless patients that can precede relapse." Secondly, prophylaxis is costly. If standard maintenance doses of cimetidine were sufficient to

\section{Should patients change non-steroidal anti-inflammatory drugs?}

Appreciable differences are found among different non-steroidal anti-inflammatory drugs in the rate of 
Controversies in

\section{Therapeutics}

prevent half of the bleeds associated with non-steroidal anti-inflammatory drugs occurring once every 500 patient years the cost for each life saved would be around $£ 1 \mathrm{~m}$. Curtailed prescribing of non-steroidal anti-inflammatory drugs and expanded programmes of joint replacement may be a better bargain.

Should every patient taking non-steroidal anti-inflammatory drugs have endoscopy?

Suspected ulcers should be confirmed endoscopically and monitored for recurrence because so many are silent. Likewise, when treatment is started endoscopy should be performed, but this is impracticable with the current amount of prescribing of non-steroidal antiinflammatory drugs.

\section{Conclusion}

Non-steroidal anti-inflammatory drugs cause significant gastrointestinal morbidity and mortality. Most patients presenting with bleeding have not seen a rheumatologist, are not taking non-steroidal antiinflammatory drugs for an inflammatory polyarthritis, and probably do not need them.' For those who do need the drugs the limited available evidence would convince many prescribers (though not yet the regulatory authorities ${ }^{23}$ ) that such drugs can be continued during the healing of ulcers. Strategies for prophylaxis are less clear and more pragmatic (box). Meanwhile, some patients with rheumatoid arthritis might inadvertently be receiving prophylaxis as sulphasalazine protects cells of the stomach in animal studies..$^{2+}$

Since this article was written misoprostol has become available for prophylaxis of gastric ulcers in patients taking non-steroidal anti-inflammatory drugs and data have been published suggesting that ranitidine may prevent duodenal ulcers. ${ }^{2}$

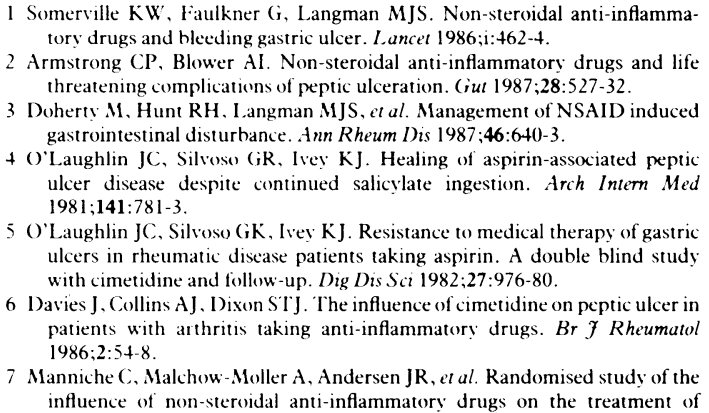

7 Manniche C, Malchow-Moller A, Andersen JR, et al. Randomised study of the influence of non-steroidal anti-inflammatory drugs on the treatment of peptic ulcer in patients with rheumatoid arthritis. Gut 1987:28:226-9.

\section{Prophylactic strategies \\ Before an ulcer develops}

Avoid non-steroidal anti-inflammatory drugs when there is no joint disease, joint disease is inactive, and arthritis is mild, especially in elderly women

Remember dyspepsia is not a reliable indicator of ulceration

\section{When an ulcer develops}

Confirm endoscopically

Stop treatment with non-steroidal

anti-inflammatory drugs if possible

Continue treatment if necessary, adding an ulcer healing agent

\section{When an ulcer has healed}

- Consider endoscopic surveillance even with maintenance treatment as relapse is often silent.

8 Agrawal N, Tulane U, Roth S, et al. Misoprostol coadministration heals aspirin induced gastric lesions in rheumatoid arthritis patients. Gastroentero$\log v 1987 ; 92: 1290$.

9 Rossi AC, Hsu JP, Faich GA. Ulcerogenicity of piroxicam: an analysis of spontaneously reported data. $\mathrm{Br}$ Med f 1987;294:147.

10 Committee on Safety of Medicines. Non-steroidal anti-inflammatory drugs and serious gastrointestinal adverse reactions. Br Med f 1986;292:1190-1.

11 O'Brien JD, Burnham WR. Bleeding from peptic ulcers and use of nonsteroidal anti-inflammatory drugs in the Romford area. Br Med f 1985;291 1609-10.

12 Jorde R, Bostad L, Burhol PG. Asymptomatic gastric ulcer: a follow-up study in patients with previous gastric ulcer disease. Lancel 1986;:1119.

13 Penston JG, Wormsley KG. Long term treatment of duodenal ulcers. Gastroenterologv 1988;94:A349.

14 Rothe SH. Bennett RE, Mitchell CS, Hartman RJ. Cimetidine therapy in nonsteroidal anti-inflammatory drug gastropathy. Double-blind long-term valuation. Arch Intern Med 1987;147:1798-801

15 Patrono C, Ciabattoni G, Badrignani P, et al. Clinical pharmacology of platelet cyclo-oxygenase inhibition. Circulation 1985;72:1177-84

16 Wheatley KE, Poxon VA, Dykes PW, Keighley MRB. Intragastric fibrinolysi in bleeding peptic ulcer disease. Gut 1987;28:A1402.

17 Daneshmend TK. Stein AG, Bhaskar NK, Hawkey CJ. Abolition by omeprazole of aspirin-induced gastric mucosal injury in humans. Gut 1988;29:A1+42.

18 Bigard MA, Isal JP. Complete prevention by omeprazole of aspirin induced gastric lesions in healthy subjects. Gut 1988;29:A712.

19 Hogden CAM, Schanker LS, Toco DJ, Brodie BB. Absorption of drugs from the stomach. II. The human. $\mathcal{A}$ Pharmacol Exp Ther 1957;120:540-5.

20 Lanza FL, Aspinall RL, Swabb EA, Davis RE, Rack MF, Rubin A. A doubleblind placebo controlled endoscopic comparison of the cytoprotective effects of misoprostol and cimetidine on tolmetin induced gastric mucosal injury. of misoprostol and cimetidine
Gastroenterologv 1987;92:1491.

21 Graham DY, Smith JL. Aspirin and the stomach. Ann Intern Med 1986;104 390-8

22 Penston J, Carter D, Wormsley KG. Recurrence of duodenal ulcers during maintenance treatment. Gut 1987;28:A1375.

23 Anonymous. FDA criticises SK\&F's Tagamet promotion. Script 1988 July $16: 16$

$24 \mathrm{Cho} \mathrm{CH}, \mathrm{Ogle} \mathrm{CW}$, Sevilla EL. The protective effects of sulphasalazine against ethanol-induced gastric damage in rats. Br f Pharmacol 1987;92:31-7.

25 Ehsanullah RSB, Page MC, Tildesley G, Wood JR. Prevention of gastrointestinal damage induced by non-steroidal anti-inflammatory drugs: controlled trial of ranitidine. Br Med $\mathcal{F}$ 1988;297:1017-21.

\section{Michael Doherty \\ continued from page 176}

have also been advocated. ' Such considerations, based on accurately assessing the patient, may avoid altogether or end treatment with non-steroidal anti-inflammatory drugs.

\section{Whether to start treatment}

Controversy, however, surrounds continuing or starting treatment with non-steroidal anti-inflammatory drugs in patients with current or past peptic ulcer disease. There are four principal concerns.

Firstly, should non-steroidal anti-inflammatory drugs be given at all? Even after considering the above, some patients with chronic locomotor symptoms find non-steroidal anti-inflammatory drugs undeniably beneficial, and their quality of life is considerably diminished when they are withdrawn. Although the Committee on Safety of Medicines states that non- steroidal anti-inflammatory drugs should not be given to patients with active peptic ulceration, ${ }^{22}$ such official advice, predominantly reflecting expectation bias, ${ }^{23}$ would result in withdrawal of such drugs in up to $30 \%$ of patients with chronic rheumatic disease. ${ }^{16}$ The few studies available, however, show that continuing treatment with the drugs little influences the rates of healing obtained with conventional anti-ulcer treatment ${ }^{162+27}$ : indeed, ulcers may heal spontaneously despite continuing non-steroidal anti-inflammatory drugs. ${ }^{28}$ If appropriate non-steroidal anti-inflammatory drugs should therefore still be considered.

Secondly, which non-steroidal anti-inflammatory drug should be prescribed? Salicylate and indomethacin should probably be avoided in peptic ulceration because of their additional direct mucosal toxicity ${ }^{2-4} 6$ (in the United Kingdom salicylate is rarely prescribed for arthritis anyway, and indomethacin should be 